\title{
VLTI/AMBER observations of the binary $B[e]$ supergiant HD 327083
}

\author{
H. E. Wheelwright ${ }^{1,2}$, W. J. de $\mathrm{Wit}^{3}$, R. D. Oudmaijer ${ }^{2}$, and J. S. Vink ${ }^{4}$ \\ ${ }^{1}$ Max-Planck-Institut für Radioastronomie, Auf dem Hügel 69, 53121 Bonn, Germany \\ e-mail: hwheelwright@mpifr-bonn.mpg.de \\ 2 School of Physics and Astronomy, University of Leeds, Leeds LS2 9JT, UK \\ 3 European Southern Observatory, Alonso de Cordova 3107, Vitacura, Santiago, Chile \\ 4 Armagh Observatory, College Hill, Armagh BT61 9DG, Northern Ireland
}

Received 25 July 2011 / Accepted 4 December 2011

\begin{abstract}
HD 327083 is a luminous B type star which exhibits emission lines and an infrared excess and is therefore classified as a supergiant $\mathrm{B}[\mathrm{e}]$ star. In addition, the star is the primary of a close binary system. It is not clear whether the B[e] behaviour of HD 327083 is related to its binarity or its evolutionary state. Here we address this issue by studying its circumstellar environment with high spatial resolution. To this end, we have observed HD 327083 with the VLTI and AMBER in the medium resolution $K$-band setting. ${ }^{13} \mathrm{CO}$ bandhead emission is detected, confirming HD 327083 is a post-main sequence object. The observations spatially resolve the source of the NIR continuum and the $\mathrm{Br} \gamma$ and $\mathrm{CO}$ line emission. In addition, differential phase measurements allow us to probe the origin of the observed $\mathrm{Br} \gamma$ emission with sub-mas precision. Using geometrical models, we find that the visibilities and closure phases suggest that the close binary system is surrounded by a circum-binary disk. We also find that in the case of the binary HD 327083, the relative sizes of the continuum and $\mathrm{Br} \gamma$ emitting regions are different to those of a single supergiant $\mathrm{B}[\mathrm{e}]$ star where the standard dual outflow scenario is thought to apply. These findings are consistent with the hypothesis that the mass loss of HD 327083 is related to its binary nature.
\end{abstract}

Key words. techniques: high angular resolution - techniques: interferometric - stars: early-type - stars: emission-line, $\mathrm{Be}-$ stars: mass-loss - stars: individual: HD 327083

\section{Introduction}

Massive stars play a pivotal role in many areas of astrophysics. Consequently, it is important to understand their evolution and how they interact with their surroundings. In turn, understanding the evolution of massive stars requires knowledge of how they lose mass and how this changes with evolutionary state (see e.g. Puls et al. 2008). Furthermore, constraining the geometry of massive star outflows is of great importance for massive star evolution models that include stellar rotation, especially with respect to the removal of angular momentum. Supergiant $\mathrm{B}[\mathrm{e}]$ $(\mathrm{sgB}[\mathrm{e}])$ stars are key objects in this regard as they are massive objects in a late evolutionary stage which also exhibit signs of enhanced mass loss (see e.g. Lamers et al. 1998). However, the mechanism responsible for the mass loss of $\mathrm{sgB}[\mathrm{e}]$ stars is still not fully understood.

The primary characteristics of $\mathrm{sgB}[\mathrm{e}]$ stars are an optical spectrum with prominent emission lines and an excess of infrared continuum emission. Typical emission lines include: Balmer lines; low excitation lines of metals such as FeII; and forbidden lines of FeII and OI. The low excitation emission lines are generally relatively narrow $\left(\sim 10 \mathrm{~s}^{\text {of }} \mathrm{km} \mathrm{s}^{-1}\right)$. Typically, $\mathrm{sgB}[\mathrm{e}]$ stars do not exhibit absorption features in the optical, but do display absorption lines due to ions such as Sirv and Civ in the UV. These features are high excitation lines, and are generally much broader than the low excitation emission lines (e.g. $1000 \mathrm{~s}$

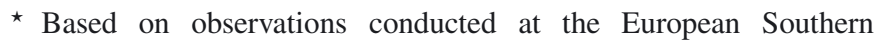
Observatory, Paranal, Chile, which were obtained as part of the program 383.C-0166. of $\mathrm{km} \mathrm{s}^{-1}$ ). As a result, the spectra of $\mathrm{sgB}[\mathrm{e}]$ have a hybrid appearance.

To explain these characteristics, Zickgraf et al. (1985) proposed a model in which $\mathrm{sgB}[\mathrm{e}]$ stars drive two distinct outflows: a fast wind in the polar regions and a slower, and denser outflow in the equatorial region. In this scenario, the narrow line emission of $\mathrm{sgB}[\mathrm{e}]$ stars originates in the dense equatorial wind while the broad absorption lines trace the fast polar wind. This model reproduces the range of line profiles exhibited by $\operatorname{sgB}[\mathrm{e}]$ stars but the origin of the equatorial outflow is still uncertain (see e.g. Puls et al. 2008). It has been proposed that when stars become cooler than $\sim 25000 \mathrm{~K}$, an increased Fe opacity might lead to bi-stable outflows in the equatorial direction (Pelupessy et al. 2000). Alternatively, rapid rotation and wind compression (see e.g. Bjorkman \& Cassinelli 1993; Curé et al. 2005) may also contribute to equatorial enhancements. These models need to be tested against observational constraints, of which there are currently few examples.

Regardless of its origin, is generally thought that this dense, disk-like equatorial outflow contains warm dust which is responsible for the infrared excess of $\mathrm{sgB}[\mathrm{e}]$ stars (see e.g. Domiciano de Souza et al. 2011). However, it has been shown that simple models of the dust emission from equatorial disks struggle to simultaneously reproduce the spectral energy distribution of the $\mathrm{sgB}[\mathrm{e}]$ star R126 in the optical/near infrared (NIR) and the mid infrared (Porter 2003). As a result, it has been suggested that free-free and free-bound emission from the polar wind can also contribute to the infrared excess of $\operatorname{sgB}[\mathrm{e}]$ stars 
(Kraus et al. 2007). Consequently, understanding the geometry traced by the NIR excess of $\operatorname{sgB}[\mathrm{e}]$ stars is not trivial.

Optical interferometry is one of the few techniques that can probe the environments of $\operatorname{sgB}[\mathrm{e}]$ stars to study their circumstellar geometries in detail. For example, Domiciano de Souza et al. (2007) used the VLTI to study the $\mathrm{sgB}[\mathrm{e}]$ star CPD $-57^{\circ} 2874$. They found that the sizes of the observed NIR continuum, Br $\gamma$ emission and mid infrared (MIR) continuum are different. Furthermore, they also found that the orientation of the near and mid infrared emission regions on the sky differs. The MIR emission was later shown to trace a dusty disk-like structure (Domiciano de Souza et al. 2011). These findings are broadly consistent with the dual outflow scenario of Zickgraf et al. (1985). However, these observations do not constrain the origin of the equatorial outflow. In a more recent use of the VLTI, Millour et al. (2009) used the AMBER instrument to reconstruct images of the $\mathrm{sgB}[\mathrm{e}]$ candidate HD 87643 with high spatial resolution. The images reveal a previously undetected binary companion. Millour et al. (2009) speculate that the complex circumstellar environment of the star has been shaped by binary interactions, suggesting binarity may play a role in the $\operatorname{sgB}[\mathrm{e}]$ phenomenon.

Despite the progress made with such observations, a general understanding of $\mathrm{sgB}[\mathrm{e}] \mathrm{stars}$ has yet to emerge. This is principally because the number of objects studied with the required spatial and spectral resolution is still very small. To increase this sample, and thus address the uncertainty regarding the $\operatorname{sgB}[\mathrm{e}]$ phenomenon, we present VLTI/AMBER observations of an additional sgB[e] star, HD 327083 (ALS 3992, CPD-40 7757, MWC 873, He 3-1359). This object is a Galactic $\operatorname{sgB}[\mathrm{e}]$ candidate (Kraus 2009) and is known to exhibit optical emission lines characteristic of sgB[e] stars (HI, HeII and FeII, see e.g. Carlson \& Henize 1979). In addition, it also exhibits CO overtone bandhead emission at $\sim 2.3 \mu \mathrm{m}$ (McGregor et al. 1988). This emission requires hot, dense conditions and thus could originate in the proposed equatorial outflow of $\operatorname{sgB}[\mathrm{e}]$ stars. Therefore, this emission is consistent with the object's classification as a $\operatorname{sgB}[\mathrm{e}]$ star (see Kraus 2009).

Lopes et al. (1992) suggest that HD 327083 has an intrinsic luminosity of $10^{6} L_{\odot}$, making it one of the most luminous objects in the Galaxy. This luminosity was calculated for a distance of $5 \mathrm{kpc}$, which Lopes et al. (1992) estimate from the equivalent width of the $\mathrm{Na}$ I lines in the object's spectrum. This high value is subject to a factor of 2 error, as discussed in Millour et al. (2009). Also, as noted by Miroshnichenko et al. (2003), the interstellar $\mathrm{Na}$ I lines of this object are saturated, resulting in an overestimation of the distance to it. Based on the radial velocity of interstellar features in the object's spectrum, these authors suggest it is located in the Sagittarius spiral arm and thus assign a distance of $d=1.5 \pm 0.5 \mathrm{kpc}$. We adopt this distance. At this distance, the luminosity will decrease by an order of magnitude, but is still sufficient to satisfy the criterion of Lamers et al. (1998) for classification as a $\mathrm{sgB}[\mathrm{e}]$ star.

Currently, the reason for the B[e] behaviour of HD 327083 is still uncertain. Based on optical spectroscopy and a NLTE model of an expanding atmosphere, Machado \& de Araújo (2003) suggest that the object may be close to the luminous blue variable phase. In the only other in-depth study of this object, Miroshnichenko et al. (2003) detected an unresolved binary companion via radial velocity variations. These authors suggest that the system is close enough to interact. In this case, the circumstellar material evidenced by the object's infrared excess may be the result of binary interactions, as proposed for lower luminosity B[e] stars (see Miroshnichenko 2007). Consequently, the evolutionary state of HD 327083 is still a matter of debate and it is not clear whether it conforms with the standard $\operatorname{sgB}[\mathrm{e}]$ scenario or not. To rectify this, we have observed HD 327083 with the VLTI to probe the source of the NIR line and continuum emission on milli-arcsecond (mas) scales and constrain its circumstellar geometry and mass loss.

This paper is structured as follows. Section 2 presents the VLTI and AMBER observations, in addition to the data reduction process used. We then present the results in Sect. 3 and discuss their implications in Sect. 4. Finally, we conclude the paper in Sect. 5.

\section{Observations and data reduction}

HD 327083 was observed with the VLTI and AMBER (see Petrov et al. 2007) on the 13th and 14th of April 2009. During observations on the 13th, light from the unit telescopes UT1, UT2 and UT3 was combined while on the 14th the UT2, UT3 and UT4 telescopes were used. The fringe tracker FINITO was used and the exposure times were $187 \mathrm{~ms}$ on the 13 th and $300 \mathrm{~ms}$ on the 14 th. On the 13 th, 1000 spectrally dispersed interferograms were recorded while 1800 such frames were recorded the following night. The data were obtained using the medium resolution $K$ setting which has a spectral resolution of $R=1500$ or $\sim 200 \mathrm{~km} \mathrm{~s}^{-1}$. The use of FINITO allowed us to record the entire spectral range and thus we observed both $\mathrm{Br} \gamma$ emission at $2.16 \mu \mathrm{m}$ and $\mathrm{CO}$ bandhead emission at $\sim 2.3 \mu \mathrm{m}$. A $\log$ of the observations is presented in Table 1 and Fig. 1 displays the projected baselines for the observations of HD 327083. While the use of FINITO can bias the final visibilities, the observations were performed before the FINITO tracking information was recorded and thus we cannot assess this possibility.

Data reduction was performed with the amdlib software (version 3, see Tatulli et al. 2007; Chelli et al. 2009). The standard data reduction procedure, i.e. determining the pixel-to-visibilitymatrix and converting the observed fringe patterns to measurements of the coherent flux, was followed. The data were then subject to frame selection in which the frames were ranked based on their fringe signal-to-noise ratio (SNR) and the best 5 per cent were selected. We found that retaining more than 5 per cent of the frames resulted in inconsistent visibilities for two baselines with similar lengths and position angles (UT1-UT2 on the 13th and UT2-UT3 on the 14th). Therefore, we adopted a selection criterion of the best 5 per cent of frames. The difference between visibilities generated with a selection criterion of 5 and 20 percent was generally less than 0.05 for other baselines, and was typically comparable to the uncertainties.

The small selection criterion resulted in relatively noisy phase information. Therefore, differential phases were calculated with a 50 per cent selection criterion. Even with this enhanced selection rate, the closure phase measurements observed with the UT1-UT2-UT3 configuration exhibited large uncertainties. To reduce the uncertainties, these measurements were recalculated with a selection rate of 80 per cent. The observations with the UT2-UT3-UT4 configuration reveal a non-zero closure phase. This exhibits a slight trend with the frame selection rate, decreasing at high frame selection rates. To minimise this effect, we recalculated these measurements with a frame selection rate of 10 per cent.

Observations of standard stars (typically with diameters of $\sim 1$ mas) were used to create the visibility transfer function. This was done by dividing the visibilities of the standards at each spectral bin by the visibility of a uniform disk with a diameter 
Table 1. Observations of HD 327083.

\begin{tabular}{lcccc}
\hline \hline Date & \multicolumn{2}{c}{ Baseline } & PA & SNR \\
& $($ UTs $)$ & $(\mathrm{m})$ & $\left.{ }^{\circ}\right)$ & $($ mean $)$ \\
\hline $13 / 04 / 09$ & UT1-UT2 & 52.3 & 36.1 & 1.9 \\
$13 / 04 / 09$ & UT2-UT3 & 42.8 & 51.3 & 4.2 \\
$13 / 04 / 09$ & UT3-UT1 & 94.2 & 42.9 & 0.9 \\
$14 / 04 / 09$ & UT2-UT3 & 46.5 & 32.0 & 6.1 \\
$14 / 04 / 09$ & UT3-UT4 & 59.6 & 100.2 & 4.1 \\
$14 / 04 / 09$ & UT4-UT2 & 88.1 & 70.8 & 1.6 \\
\multicolumn{7}{c}{ 13/04/09 } & Standard stars: HD 194013 and HD 161068 \\
$14 / 04 / 09$ & Standard stars: HD 109963 and HD 161068 \\
\hline
\end{tabular}

Notes. Column 1 contains the dates of the observations, Cols. 2-4 describe the baselines used and Col. 5 lists the average frame signal to noise ratio (SNR) for each baseline. The adopted diameters of the calibrators were HD 194013: $1.1132 \pm 0.091$, HD 161068: $1.43972 \pm$ 0.01975, HD 109963: $0.784989 \pm 0.01025$ mas.

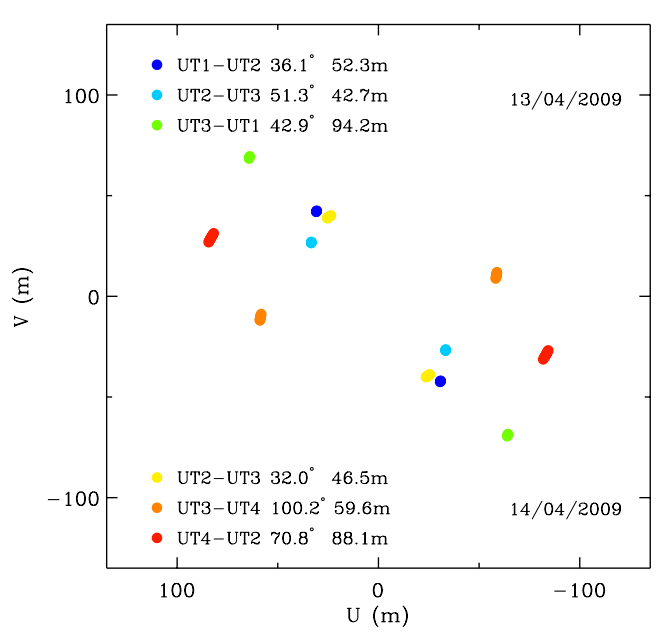

Fig. 1. The projected baselines of the observations of HD 327083.

equal to that of the standards. Standard stars were observed before and after the observations of HD 327083. This allowed the behaviour of the transfer function with time to be constrained. Nonetheless, there could be a systematic uncertainty in the transfer functions of the order of 0.05 due to temporal variations. The transfer functions were generally of the order of 0.1 indicating poor fringe contrast, perhaps as a result of telescope vibrations.

The standard stars used are late type giants with spectral types of K3III, G8III/IV and K4/5 III ${ }^{1}$. Such stars exhibit photospheric absorption features due to molecules. For example, both $\mathrm{G}$ and $\mathrm{K}$ type giants exhibit absorption due to $\mathrm{CO}$ at $\sim 2.3 \mu \mathrm{m}$. The visibilities of the standard star HD 161068 change over the wavelengths corresponding to the $\mathrm{CO}$ absorption. This implies that we begin to resolve the outer layers of the stellar surface. To avoid introducing artifacts in the science data, the transfer functions were fit with low order polynomial functions which were then used to calibrate the science data.

The spectrum of HD 161068 was then used to remove telluric absorption features from the spectrum of HD 327083. To achieve this without introducing artifacts in the final spectra, the intrinsic $\mathrm{CO}$ bandhead absorption features in the spectrum of HD 161068 also had to be removed. This was done following the example of Tatulli et al. (2008). A template spectrum was created

\footnotetext{
1 from SIMBAD: http://simbad.u-strasbg.fr/simbad/
}

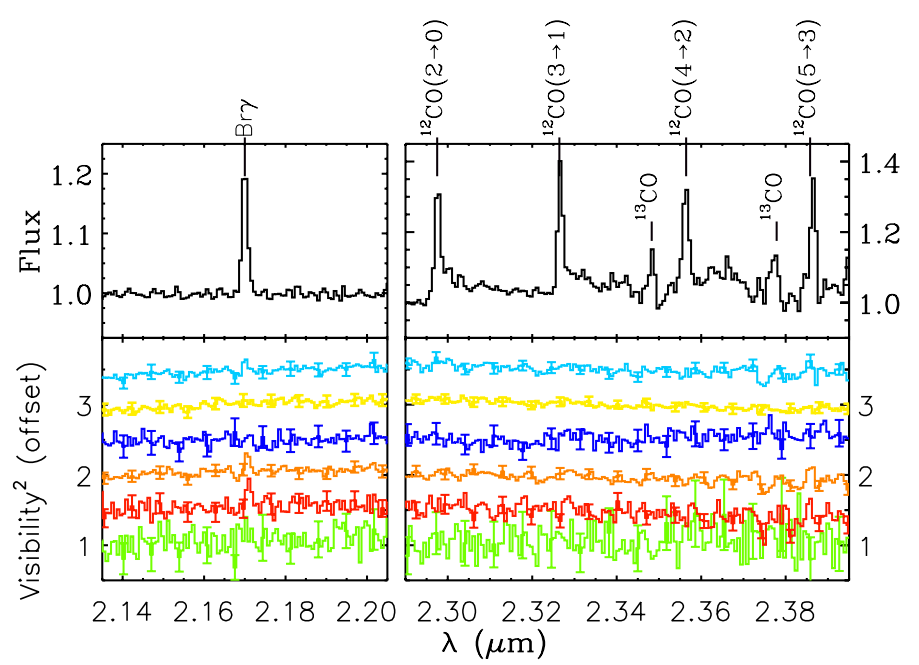

Fig. 2. The average spectrum and differential visibilities of HD 327083. The upper panel presents the spectrum featuring $\mathrm{Br} \gamma$ and $\mathrm{CO}$ emission. The visibilities are shown offset (by an integer times 0.5 ) to aid comparison. They are shown to scale in Fig. 3.

by averaging spectra of stars with a similar spectral type in the database of Wallace \& Hinkle (1997, HD 62721, HD 70272 and HD 164058). The average spectrum was then multiplied by the slope of a model SED (taken from Castelli \& Kurucz 2004), which was computed for parameters appropriate for the spectral type of the standard. The resultant spectrum was smoothed to match the resolution of the observations and was then used to remove the intrinsic absorption features of the standard spectra.

\section{Results}

The observations of HD 327083 are presented in Figs. 2 and 3. The spectrum, displayed in Fig. 2, exhibits $\mathrm{Br} \gamma$ emission and $\mathrm{CO}$ overtone bandhead emission at $\sim 2.3 \mu \mathrm{m}$. While the ${ }^{12} \mathrm{CO}$ overtones dominate the emission, the spectrum also exhibits two features at $\sim 2.35$ and $\sim 2.37 \mu \mathrm{m}$ that are identified as ${ }^{13} \mathrm{CO}$ emission (these can also be seen in the lower resolution data of McGregor et al. 1988). This is consistent with the identification of HD 327083 as a star in an advanced evolutionary state and confirms the object's classification as a $\operatorname{sgB}[\mathrm{e}]$ star (see Kraus 2009; Liermann et al. 2010). The Br $\gamma$ emission has a Full-Width-at-Half-Maximum of $\sim 220 \mathrm{~km} \mathrm{~s}^{-1}$, indicating that it is marginally resolved (in the spectral domain).

The visibilities of HD 327083 as a function of wavelength and spatial frequency are presented in Figs. 2 and 3 respectively. The squared visibilities range from $\sim 0.05$ to $\sim 0.4$. Consequently, we find that the source of the NIR continuum is resolved on all baselines. We note that a slight increase in visibilities is detected across the $\mathrm{Br} \gamma$ emission at certain position angles $\left(50-100^{\circ}\right.$, see Fig. 2). No clear change is observed in the visibilities across the $\mathrm{CO}$ bandhead emission. There is a suggestion of an increase in visibilities over the 4th bandhead, but as this is not replicated over the adjacent bandhead, we do not treat this as a robust detection. The increase in visibilities indicates that in certain directions, the $\mathrm{Br} \gamma$ emitting region is less extended than the region responsible for the continuum emission. The $\mathrm{Br} \gamma$ and $\mathrm{CO}$ emission features are of a comparable strength, so this difference in behaviour is unlikely to be due to a difference in contrast with the continuum. Therefore, the visibilities also indicate that the spatial distribution of the $\mathrm{CO}$ and the $\mathrm{Br} \gamma$ emission is different. 

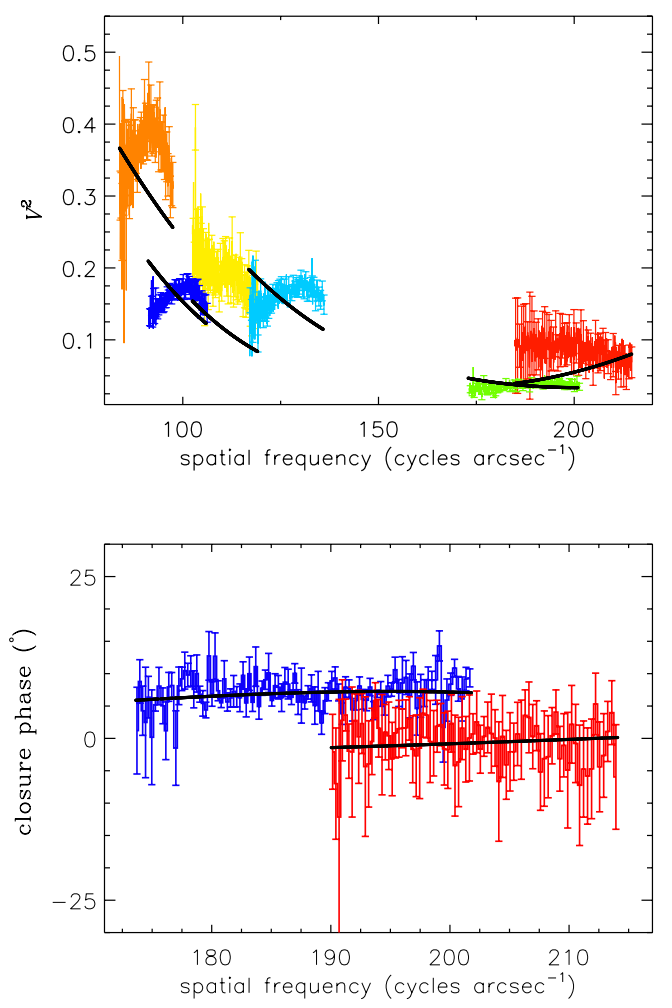

Fig. 3. The visibilities and closure phases of HD 327083 as a function of spatial frequency. The closure phase measurements have been re-binned by a factor of 5. Closure phases observed with the UT1-UT2-UT3 configuration at spatial frequencies below 190 cycles per arcsecond have been omitted as these exhibited a large scatter. The solid lines represent the best fitting model discussed in Sect. 3.1.1.

The average differential phase measurements over the $\mathrm{Br} \gamma$ emission are shown in Fig. 4. Changes in phase are associated with a displacement of the photocentre via the equation:

$p=-\frac{\Delta \phi}{2 \pi} \frac{\lambda}{B}$

where $\lambda$ and $B$ are the wavelength and projected baseline associated with the observation, $p$ is the projected photocentre displacement and $\Delta \phi$ is the differential phase signature (see e.g. Lachaume 2003). The sign of the phase shift determines the direction of the photocentre displacement. We re-reduced the AMBER data of Benisty et al. (2010), who map the differential phase signature of Z CMa onto the direction of a known jet, and find our treatment of the differential phases is consistent with theirs. The final photocentre shift per spectral channel is calculated by forming a system of linear equations from the linear shifts in the directions of the individual baselines.

A shift in phase corresponding to a photo-centre shift of the order of 0.1 mas can be observed over the $\mathrm{Br} \gamma$ emission, particularly in the data obtained with the UT2-UT3-UT4 configuration. The one-sided nature of the differential phase signature indicates that the direction of the photo-centre shift is constant. This is shown in Fig. 4 where the displacements are shown on the plane of the sky. The excursion in one direction is contrary to the case of a rotating disk where the blue and red shifted photo-centres are located on opposite sides of the continuum. No such signature is observed over the CO bandhead emission. This indicates that it has a more symmetrical and/or more compact distribution.

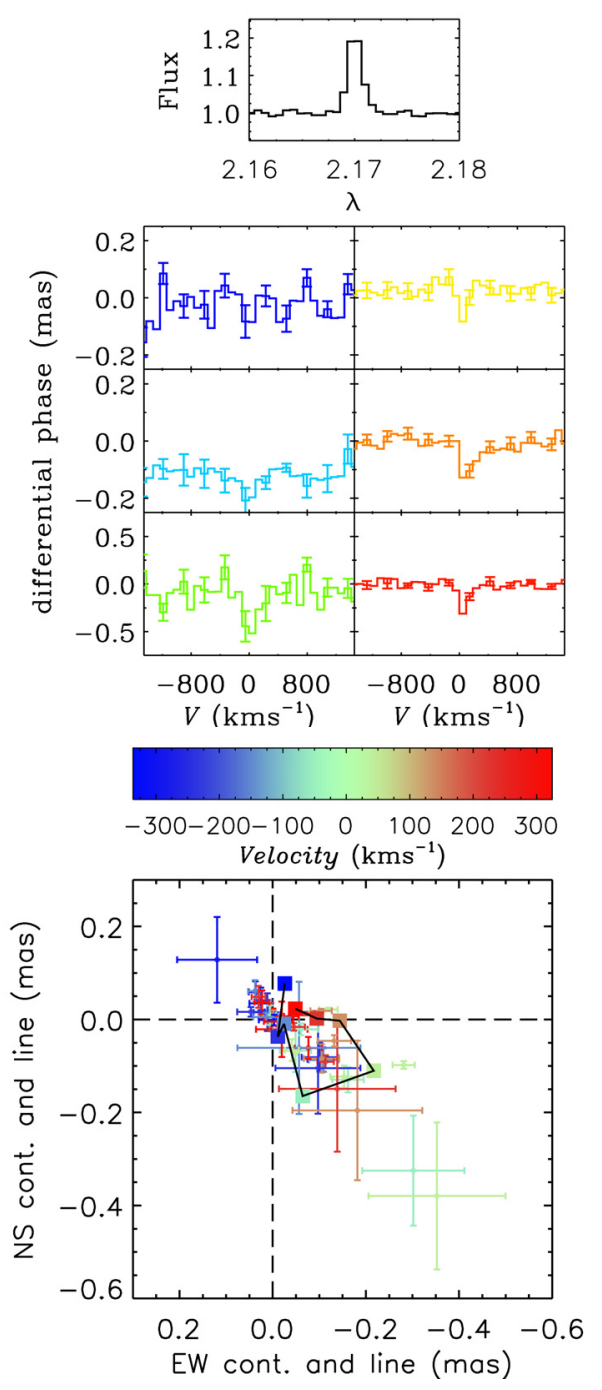

Fig. 4. The average differential phases over the $\mathrm{Br} \gamma$ emission for each of the six baselines used and the calculated photo-centre shift. The calculated total shift per spectral channel is marked by the square points.

Turning to the closure phase measurements, also shown in Fig. 3, the averaged measurement for each configuration reveals a closure phase that is essentially constant with wavelength (spatial frequency). In the case of the UT1-UT2-UT3 configuration, the average closure phase is $\sim 0^{\circ}$ while in the case of the UT2-UT3-UT4 configuration it is approximately $7^{\circ}$. The closure phases of the calibrators do not exhibit this signature and thus it is unlikely to be due to a systematic effect. Instead, the nonzero closure phase observed suggest the presence of an asymmetric flux distribution, which is expected in the case of a binary system.

\subsection{Modelling the observations}

\subsubsection{Continuum visibilities and closure phases}

To investigate the circumstellar environment traced by the observations, we fit the visibilities and closure phase measurements with a simple geometrical model.

The model is constructed to represent the binary system detected by Miroshnichenko et al. (2003). Specifically, the binary components are treated as uniform disks with radii of 27 and $86 R_{\odot}$, diameters of 0.17 and 0.53 mas at $1500 \mathrm{pc}$, respectively. 


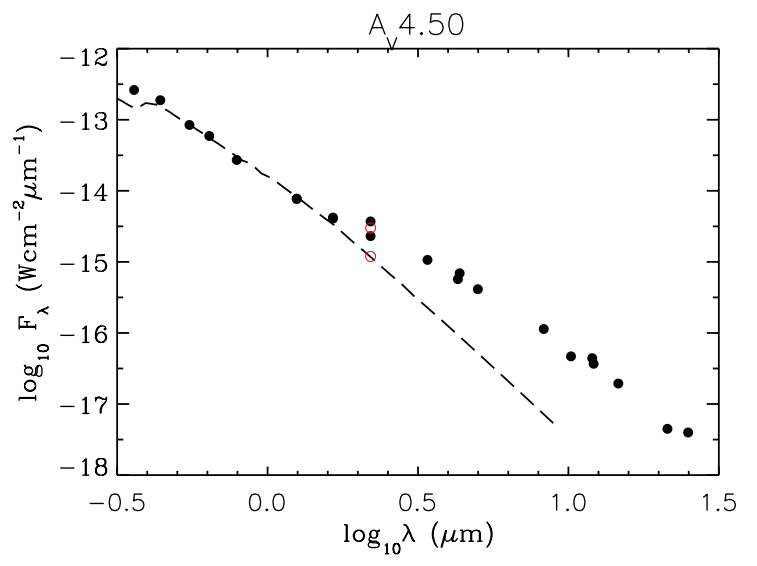

Fig. 5. The de-reddened SED of HD 327083. The dashed line marks the model SED of the proposed binary system, scaled to fit the visible SED. The model SEDs are taken from Castelli \& Kurucz (2004).

A flux ratio of 3.5 was estimated from the visual brightness ratio and model SEDs (taken from Castelli \& Kurucz 2004) appropriate for the stellar properties reported by Miroshnichenko et al. (2003).

The observed visibilities are unlikely to be fit by a model of a binary system alone. Such a scenario would result in a sinusoidal visibility signature, and the low visibilities observed clearly indicate an extended component. The system also contains circumstellar material, as evidenced by the infrared excess. Therefore, we add an additional component to the binary model. Determining the circumstellar contribution to the $K$-band flux requires the SED of HD 327083. Using the data presented in: Miroshnichenko et al. (2003), Cutri et al. (2003), Egan et al. (2003) and Beichman et al. (1988), the SED of HD 327083 was constructed (see Fig. 5). We adopted a value of $A_{V}=4.5$ to de-redden the final SED. Based on optical colour indices, Miroshnichenko et al. (2003) estimate a value of $A_{V}=5.6 \pm 0.5$. However, the value adopted provides a closer match between the UV flux and the model binary SED. The final estimate for the circumstellar contribution to the $K$-band flux is 1.5 times that of the binary. If the reddening is greater than the value adopted, this is reduced and vice versa.

We initially represented the circumstellar material by an elliptical Gaussian function centred on the position of the primary component. Fitting the visibilities only, the best fitting model resulted in $\chi^{2}=4.4$ and featured a Gaussian that was extended along a PA of $\sim 160^{\circ}$ with a major FWHM of approximately 10 mas. As a result, it is significantly more extended than the expected binary separation, which is limited to a maximum of several mas by radial velocity variations. Therefore, it would appear that the circumstellar material is not truncated by the presence of the companion. This presents two possibilities: either the NIR excess traces a bi-polar outflow from the primary that is oriented perpendicular to the binary system or the NIR excess originates in a circum-binary disk.

The zero-closure phase observed with the UT1-UT2-UT3 array, which is an approximately linear configuration with a PA of $40^{\circ}$, implies that the binary PA is $\sim 130^{\circ}$. As a result, taking into account the maximum expected binary separation, it appears the binary system is situated interior to the source of the NIR excess. This is more reminiscent of a circum-binary disk than an outflow. Therefore, we now model the circumstellar material as a circum-binary disk, which we approximate as an elongated, uniform ring. We note that the material traced by the line emission and the NIR continuum is not necessarily distributed identically.

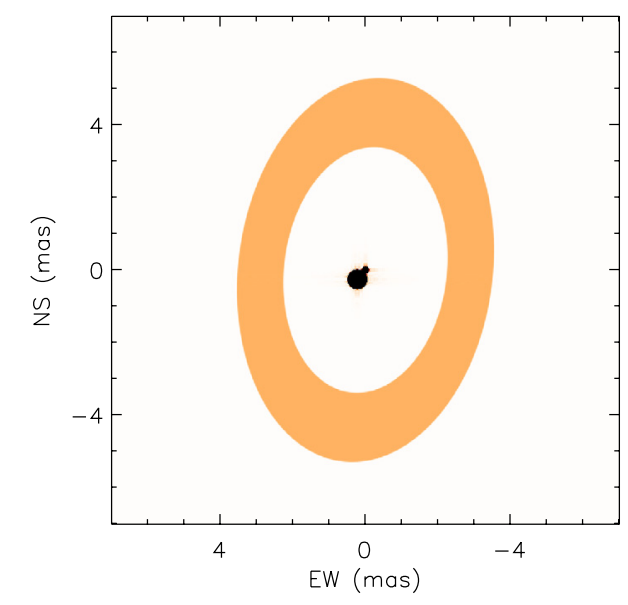

Fig. 6. The image of the best fitting model. North is towards the top of the page and East is to the left. The image has been logarithmically scaled.

Therefore, to begin with, we consider only the continuum light and fit the visibilities over the entire spectral range. The visibilities over the $\mathrm{Br} \gamma$ and $\mathrm{CO}$ emission are discussed in the next section.

The free parameters of the final model were: the binary separation and position angle (PA), the radius and width of the uniform ring, its elongation and its PA. To fit the observed visibilities with the model, we used LITpro ${ }^{2}$ (Lyon Interferometric Tool prototype), a piece of software developed by the Jean-Marie Mariotti Center (JMMC, Tallon-Bosc et al. 2008). LITpro fits geometrical models to interferometric observables by using the Levenberg-Marquardt algorithm to minimise $\chi^{2}$. Since the radial velocity variations of HD 327083 suggest the maximum binary separation is of the order of several AU, the separation of the binary model was limited to less than approximately 4.0 mas.

In an attempt to identify the global minima in $\chi^{2}$, we initialised the fitting program with 50 different sets of values for the free parameters. The best fitting model resulted in a $\chi^{2}$ value of 4.4. As can be seen in Fig. 3, this moderately high value is partly due to the inability of the binary model to reproduce the curve of the visibilities at low spatial frequencies $(\sim 100$ cycles per $\operatorname{arcsec}$ ). The close binary system cannot produce modulations in the visibilities with such a pronounced curve. This may be the signature of some additional source of flux such as localised nebulosity or an additional companion that is fully resolved at baselines of $\sim 90 \mathrm{~m}$. Further observations are planned to improve our $u, v$ coverage to investigate this. For now, we surmise that the value of $\chi^{2}=4.4$ indicates that the fit is reasonable for the model that we are using. The best fitting parameters are presented in Table 2 and the visibilities of the best fitting model are displayed in Fig. 3. Finally, the image of the best fitting model is show in Fig. 6.

\subsection{2. $\mathrm{Br} \gamma$ and $\mathrm{CO}$ visibilities}

At certain position angles, the visibilities of HD 327083 increase over wavelengths corresponding to the $\mathrm{Br} \gamma$ emission (see Fig. 2). The increase in visibilities is accompanied by a differential phase shift indicating an offset from the continuum photo-centre. The $\mathrm{CO}$ bandhead emission, which has a higher line-to-continuum

${ }^{2}$ LITpro software available at http://www . jmmc. fr/litpro 
Table 2. The best fitting parameters of the binary and elongated ring model from LITpro.

\begin{tabular}{lcc}
\hline \hline Parameter & Value & Notes \\
\hline Bin. sep & $0.34 \pm 0.01 \mathrm{mas}$ & $0.5 \mathrm{AU}, 4.0 R_{\star}$ \\
Bin. PA & $139.1 \pm 0.1^{\circ}$ & \\
Ring radius & $3.40 \pm 0.02 \mathrm{mas}$ & $5.1 \mathrm{AU}, 40.5 R_{\star}$ \\
Ring width & $1.91 \pm 0.03 \mathrm{mas}$ & $2.9 \mathrm{AU}, 22.7 R_{\star}$ \\
Ring flattening & $1.51 \pm 0.01$ & inclination $\sim 48.5$ \\
Ring PA & $173.0 \pm 0.2^{\circ}$ & \\
$\chi^{2}$ & 4.44 & \\
\hline
\end{tabular}

Notes. As discussed in Domiciano de Souza et al. (2011), the uncertainties reported by the Levenberg-Marquardt algorithm should be considered lower limits to the actual uncertainty in the best fitting parameters. The sizes in $\mathrm{AU}$ and $R_{\star}$ were calculated assuming a distance of $1500 \mathrm{pc}$.

Table 3. The visibilities over the spectral lines and the ratio of the line visibilities with those of the adjacent continuum.

\begin{tabular}{lcccccc}
\hline \hline \multirow{2}{*}{ Region } & & \multicolumn{5}{c}{$V_{\text {final }}^{2}$} \\
& UT1-2 & UT2-3 & UT1-3 & UT2-3 & UT3-4 & UT2-4 \\
\hline Br $\gamma$ & 0.20 & 0.70 & 0.27 & 0.19 & 0.37 & 0.14 \\
Br $\gamma /$ cont & 1.22 & 1.93 & 3.60 & 1.13 & 2.12 & 3.64 \\
CO & 0.23 & 0.49 & 0.07 & 0.19 & 0.19 & 0.03 \\
$\mathrm{CO} /$ cont & 1.21 & 1.25 & 0.82 & 1.13 & 1.16 & 0.79 \\
\hline
\end{tabular}

Notes. The visibilities correspond to the 6 average baselines presented in Fig. 1.

ratio, exhibits no such features. The fact that the $\mathrm{CO}$ emission is not associated with such signatures strongly suggests that they are not an artifact caused by the frame selection process. Here we investigate what geometry can recreate this behaviour.

The visibility over a spectral line can be calculated by:

$V_{\text {line }}=\frac{F_{\text {tot. }} V_{\text {tot }}-F_{\text {cont. }} V_{\text {cont. }}}{F_{\text {line }}}$

where $F_{\text {line }}=F_{\text {tot. }}-F_{\text {cont. }}$ and assuming that the phase is constant over the line (see e.g. Malbet et al. 2007). If there is a change in the differential phase of $\Phi^{\prime}$, the following equation must be used:

$$
\begin{aligned}
& V_{\text {line }}= \\
& \frac{\sqrt{\left|V_{\text {tot. }} F_{\text {tot. }}\right|^{2}+\left|V_{\text {cont. }} F_{\text {cont. }}\right|^{2}-2 V_{\text {tot. }} F_{\text {tot. }} V_{\text {cont. }} F_{\text {cont. }} \cos \Phi^{\prime}}}{F_{\text {line }}}
\end{aligned}
$$

(see e.g. Weigelt et al. 2007). There is a clear differential signature over the $\mathrm{Br} \gamma$ emission. Therefore, to calculate the visibility over this line we employ Eq. (3). The CO bandhead emission exhibits no such signature and thus we use Eq. (2) to calculate the $\mathrm{CO}$ visibilities. Since the lines are only marginally resolved in the spectral domain, we calculate the visibilities for the line peaks only. In the case of the $\mathrm{CO}$ emission, we concentrate on the 1 st bandhead alone. We list the final quantities in Table 3. The ratios of the line and continuum visibilities indicate that the line emitting regions are generally more compact that the continuum emitting region.

To estimate characteristic sizes of the regions responsible for the observed emission lines, we attempt to recreate the line visibilities presented in Table 3 with a simple model of a flattened ring. To assess the relationship between the line and continuum emission, we attempt to fit the line visibilities with the same elongation and PA as the best fitting continuum model and we vary only the size to match the line visibilities.

The increase in the visibilities over the $\mathrm{Br} \gamma$ line at PAs of $50-100^{\circ}$ clearly indicates that the line emitting region is more compact than the continuum emitting region. In addition, it also appears more compact than the $\mathrm{CO}$ emission at these position angles. This decrease in size at specific PAs cannot be recreated with the simple ring model with the same flattening and $\mathrm{PA}$ as the continuum ring. On the contrary, the $\mathrm{CO}$ visibilities can be relatively well reproduced with a family of ring models which have an inner radius in the range $\sim 0.5-2.5$ mas and a total extension (inner radius plus width) of approximately 3.5 mas (5.25 AU). This corresponds to the inner radius of the ring that fits the continuum visibilities and thus places the $\mathrm{CO}$ emission within the continuum emitting region. However, we note that without a clear visibility signature, the size of the $\mathrm{CO}$ emission is not tightly constrained. Therefore, while we find that a compact $\mathrm{CO}$ emitting region is consistent with the data, a more extended region cannot be excluded.

\subsection{A summary of the observational results}

To summarise the results, we have shown that the observations can be reproduced by a simple model of a close binary system with an additional circum-binary component. We have resolved the material responsible for the NIR excess of HD 327083 and its line emission. The continuum and CO emission appear to originate from an elongated disk-like structure that encompasses the binary system. The Br $\gamma$ emission is distributed differently and appears more compact than the continuum and $\mathrm{CO}$ emission. These results constitute the first direct constraints on the distribution of the circumstellar material associated with HD 327083 and allow a new insight into the behaviour of this object and its classification as a $\operatorname{sgB}[\mathrm{e}]$ star. This is discussed in the following section.

\section{Discussion}

Miroshnichenko et al. (2003) report that HD 327083 is a spectroscopic binary with a period of approximately 6 months and an orbital semi-major axis of $\sim 2$ AU. Our observations can be reproduced by a model of a binary system. In particular, the nonzero closure phase detected is a clear indicator of an asymmetric flux distribution which we attribute to the presence of the binary companion. This will be investigated further with multi-epoch observations.

It has been suggested that the system experiences mass transfer during phases of the orbit close to periastron (Miroshnichenko et al. 2003). If this is the case, the circumstellar material may not be mass lost from the primary via an equatorial outflow (as envisaged in the standard $\mathrm{sgB}[\mathrm{e}]$ scenario). Instead, it is possible that the material is lost from one of the components as it fills its Roche lobe. Our high spatial resolution observations resolve the circumstellar material surrounding the system and constrain the binary separation. This allows us to examine the relationship between them in order to assess the suggestion that the circumstellar material is the result of binary interactions.

The continuum visibilities and closure phases suggest that the binary is surrounded by a dusty disk. While the extent of the CO bandhead emission is not well constrained, the data are consistent with the $\mathrm{CO}$ emission being aligned with the continuum emission although less extended. CO bandhead emission originates in hot, dense gas, exactly the conditions expected 
in circumstellar disks (Wheelwright et al. 2010, and references therein). Therefore, the inferred distribution of the $\mathrm{CO}$ emission is consistent with the scenario of a gaseous disk interior to a larger, dusty disk. The inner radius of the continuum emitting ring, $\sim 40 R_{\star}$, is compatible with the expected dust sublimation radius of an early B-type star, which supports the scenario of an outer dusty disk. Turning to the $\mathrm{Br} \gamma$ emission, an increase in visibilities at certain PAs suggest a more compact and more asymmetric distribution than those of the continuum and the $\mathrm{CO}$ emission. A plausible scenario to account for this is that the Br $\gamma$ emission contains a contribution from the circumstellar environment close to the central star, where $\mathrm{CO}$ molecules cannot exist.

The differential phase signature associated with the $\operatorname{Br} \gamma$ line and the lack of a signature over the $\mathrm{CO}$ bandhead emission is consistent with the scenario in which the $\mathrm{CO}$ and $\mathrm{Br} \gamma$ emission originate in different locations. The lack of a differential phase signature over the $\mathrm{CO}$ emission is attributed to the small photo-centre offsets of $\mathrm{CO}$ emission originating in disks (see e.g. Wheelwright et al. 2010) and the limited spectral resolution of AMBER in the medium resolution mode. High spectral resolution observations are planned to assess this hypothesis. The onesided nature of the photo-centre offset over Br $\gamma$ indicates that it originates from an asymmetric region. While a scenario in which the $\mathrm{Br} \gamma$ emission emanates directly from one of the stellar components of a binary system could result in such a signature, the PA of the photocentre shift appears to differ from the inferred binary PA. Therefore, it seems that the $\mathrm{Br} \gamma$ emission traces some additional asymmetry in the environment of HD 327083. We note that the presence of the close binary companion might result in an asymmetric environment on mas scales which could explain the appearance of the differential phase signature.

To summarise, the interferometric data-set are consistent with the scenario of a circum-binary disk. Since HD 327083 is an evolved system, the circum-binary material is presumably composed of mass lost from the system. In the case of $\mathrm{sgB}[\mathrm{e}]$ stars, it is assumed that the circumstellar material is the result of intrinsic mass loss. However, here the mass loss could also be the result of binary interactions. If this is the case, binary $\mathrm{sgB}[\mathrm{e}]$ stars may represent a distinct sub-sample of the $\mathrm{sgB}[\mathrm{e}]$ population (similar to lower luminosity binary Be stars, see e.g. Miroshnichenko 2007). Here, we assess this notion by comparing these observations to similar studies of $\mathrm{B}[\mathrm{e}]$ and $\mathrm{A}[\mathrm{e}]$ objects.

Few similar studies exist. However, we note that $\mathrm{CPD}-57^{\circ} 2874$, one of the few other $\mathrm{sgB}[\mathrm{e}]$ stars studied with AMBER, is not thought to be a binary and does exhibit a different behaviour to HD 327083. In the data presented here, the visibilities increase over the $\mathrm{Br} \gamma$ line, indicating that the line emitting region is smaller than that emitting in the continuum. In the case of CPD- $57^{\circ} 2874$, the reverse is true (see Domiciano de Souza et al. 2007). We also note that the scenario of a dusty circum-binary disk with a gaseous interior is similar to the circumstellar environment of the A[e] star HD 62623 as revealed by VLTI/AMBER observations (Millour et al. 2011). As an A-type star, HD 62623 is cooler than $\operatorname{sgB}[\mathrm{e}]$ stars and is also less luminous. The currently favoured explanation of the $\mathrm{sgB}[\mathrm{e}]$ phenomenon, the bi-stability mechanism, is more effective at high luminosities (Pelupessy et al. 2000). Consequently, it is thought that the circumstellar material surrounding HD 62623 is instead the result of binary interactions (Millour et al. 2011). The similarity between the circumstellar environments of HD 62623 and HD 327083 supports the hypothesis that the B[e] behaviour of HD 327083 is the result of binary interactions rather than intrinsic mass loss.

\section{Conclusion}

This paper presents VLTI and AMBER observations of the $\mathrm{sgB}[\mathrm{e}]$ star HD 327083 . Here we list the salient findings.

- HD 327083 exhibits ${ }^{13} \mathrm{CO}$ bandhead emission, this confirms that this object is in a post-main sequence evolutionary phase.

- We spatially resolve the circumstellar environment of HD 327083 for the first time. A non-zero closure phase is observed. We attribute this to the binary companion detected via radial velocity variations.

- We find that the spatial distribution of the NIR continuum excess emission can be described as an elongated ring 10.6 mas in size that encompasses the binary system. We associate this with a dusty circum-binary disk.

- The data are consistent with the notion that the $\mathrm{B}[\mathrm{e}]$ behaviour of HD 327083 is due to binarity.

As a final remark, we note that currently few high spatial resolution observations of $\mathrm{sgB}[\mathrm{e}]$ candidates have been reported. To date, AMBER observations of three Galactic sgB[e] candidates have been published: HD 87643 (Millour et al. 2009), CPD-57²874 (Domiciano de Souza et al. 2007) and V921 Sco (Kraus et al. 2008; Kreplin et al. 2011). One of these stars is found to have a binary companion (HD 87643). With these results, the fraction of $\mathrm{sgB}[\mathrm{e}]$ candidates observed with high spatial resolution that could be interacting binaries increases to 50 per cent. This raises the possibility that binarity may play a more important role in the Galactic $\mathrm{sgB}[\mathrm{e}]$ phenomenon than previously thought. High angular resolution observations of additional $\mathrm{sgB}[\mathrm{e}]$ stars are required to assess this possibility.

Acknowledgements. The authors acknowledge the helpful and insightful comments of an anonymous referee whose suggestions helped improve the paper. This research has made use of the AMBER data reduction package of the Jean-Marie Mariotti Center ${ }^{3}$. This publication also makes use of data products from the Two Micron All Sky Survey, which is a joint project of the University of Massachusetts and the Infrared Processing and Analysis Center/California Institute of Technology, funded by the National Aeronautics and Space Administration and the National Science Foundation. Finally, this research has employed the Jean-Marie Mariotti Center LITpro service codeveloped by CRAL, LAOG and FIZEAU.

\section{References}

Beichman, C. A., Neugebauer, G., Habing, H. J., Clegg, P. E., \& Chester, T. J. 1988, Infrared astronomical satellite (IRAS) catalogs and atlases (Washinton, DC: NASA RP-1190), 1

Benisty, M., Malbet, F., Dougados, C., et al. 2010, A\&A, 517, L3

Bjorkman, J. E., \& Cassinelli, J. P. 1993, ApJ, 409, 429

Carlson, E. D., \& Henize, K. G. 1979, Vistas Astron., 23, 213

Castelli, F., \& Kurucz, R. L. 2004, in IAU Symp. p. 210, ed. N. Piskunov et al.

Chelli, A., Utrera, O. H., \& Duvert, G. 2009, A\&A, 502, 705

Curé, M., Rial, D. F., \& Cidale, L. 2005, A\&A, 437, 929

Cutri, R. M., Skrutskie, M. F., van Dyk, S., et al. 2003, 2MASS All Sky Catalog of point sources (Pasadena, CA: NASA/IPAC)

Domiciano de Souza, A., Driebe, T., Chesneau, O., et al. 2007, A\&A, 464, 81

Domiciano de Souza, A., Bendjoya, P., Niccolini, G., et al. 2011, A\&A, 525, A22

Egan, M. P., Price, S. D., Kraemer, K. E., et al. 2003, VizieR Online Data Catalog, 5114, 0

Kraus, M. 2009, A\&A, 494, 253

Kraus, M., Borges Fernandes, M., \& de Araújo, F. X. 2007, A\&A, 463, 627

Kraus, S., Hofmann, K.-H., Benisty, M., et al. 2008, A\&A, 489, 1157

Kreplin, A., Kraus, S., Hofmann, K.-H., et al. 2012, A\&A, 537, A103

Lachaume, R. 2003, A\&A, 400, 795

Lamers, H. J. G. L. M., Zickgraf, F.-J., de Winter, D., Houziaux, L., \& Zorec, J. 1998, A\&A, 340, 117

3 Available at http://www . jmmc. fr/amberdrs 
A\&A 538, A6 (2012)

Liermann, A., Kraus, M., Schnurr, O., \& Fernandes, M. B. 2010, MNRAS, 408, L6

Lopes, D. F., Damineli Neto, A., \& de Freitas Pacheco, J. A. 1992, A\&A, 261, 482

Machado, M. A. D., \& de Araújo, F. X. 2003, A\&A, 409, 665

Malbet, F., Benisty, M., de Wit, W., et al. 2007, A\&A, 464, 43

McGregor, P. J., Hyland, A. R., \& Hillier, D. J. 1988, ApJ, 324, 1071

Millour, F., Chesneau, O., Borges Fernandes, M., et al. 2009, A\&A, 507, 317

Millour, F., Meilland, A., Chesneau, O., et al. 2011, A\&A, 526, A107

Miroshnichenko, A. S. 2007, ApJ, 667, 497

Miroshnichenko, A. S., Levato, H., Bjorkman, K. S., \& Grosso, M. 2003, A\&A, 406, 673
Pelupessy, I., Lamers, H. J. G. L. M., \& Vink, J. S. 2000, A\&A, 359, 695

Petrov, R. G., Malbet, F., Weigelt, G., et al. 2007, A\&A, 464, 1

Porter, J. M. 2003, A\&A, 398, 631

Puls, J., Vink, J. S., \& Najarro, F. 2008, A\&AR, 16, 209

Tallon-Bosc, I., Tallon, M., Thiébaut, E., et al. 2008, SPIE Conf. Ser., 7013, 44

Tatulli, E., Millour, F., Chelli, A., et al. 2007, A\&A, 464, 29

Tatulli, E., Malbet, F., Ménard, F., et al. 2008, A\&A, 489, 1151

Wallace, L., \& Hinkle, K. 1997, ApJS, 111, 445

Weigelt, G., Kraus, S., Driebe, T., et al. 2007, A\&A, 464, 87

Wheelwright, H. E., Oudmaijer, R. D., de Wit, W. J., et al. 2010, MNRAS, 408, 1840

Zickgraf, F., Wolf, B., Stahl, O., Leitherer, C., \& Klare, G. 1985, A\&A, 143, 421 